\title{
PARÂMETROS COMPORTAMENTAIS DE FRANGOS DE CORTE ALIMENTADOS COM DIETAS CONTENDO DIFERENTES NÍVEIS DE VITAMINA E
}

Behavioral parameters of chicken feeded with diets containing different levels of vitamin and

Parámetros de comportamiento del pollo alimentado con dietas que contienen diferentes niveles de vitamina y

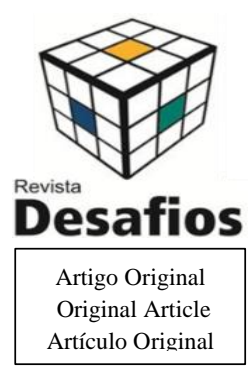

\section{Jerry Kleube Felix Monteiro Junior ${ }^{* 1}$, Mônica Calixto da Silva ${ }^{2}$, Roberta G M V Vaz ${ }^{3}$}

${ }^{1}$ Aluno do curso de Zootecnia, Universidade Federal do Tocantins, Campus de Araguaína-TO, Brasil.

${ }^{2}$ Orientadora do Curso de Zootecnia, Universidade Federal do Tocantins, Campus de Araguaína-TO, Brasil.

${ }^{3}$ Coorientadora do Curso de Zootecnia, Universidade Federal do Tocantins, Campus de Araguaína-TO, Brasil.

*Correspondência: Laboratório de Ciências, Instituto de Ensino Superior, Av. NS 15, 109 Norte, Palmas,

Tocantins, Brasil.CEP:77.010-090.e-mailartigo@artigo.com.

Artigo recebido em 09/03/2020 aprovado em 06/11/2020 publicado em 06/11/2020

\section{RESUMO}

Objetivou-se avaliar os efeitos de diferentes níveis de vitamina E na alimentação de frangos de corte sobre os parâmetros comportamentais e o consumo de ração em diferentes fases de produção. Foram utilizados 240 pintos de corte, machos, com oito dias de vida da linhagem Cobb $500^{\circledR}$. As aves foram homogeneizadas e distribuídas em delineamento inteiramente casualizado, com quatro tratamentos (50,100, 150 e 200\% das exigências de vitamina E), e seis repetições. As variáveis avaliadas foram os padrões comportamentais das aves, o consumo de ração ao longo de 24 horas e as imagens termográficas. Observou-se que os níveis crescentes de vitamina E nas dietas, não influenciaram os parâmetros comportamentais, comendo, bebendo, ócio e outras atividades. Não houve interação entre os níveis de vitamina E para as temperaturas superficiais máxima, mínima e a amplitude térmica, nos períodos da manhã e tarde. Os níveis de vitamina E nas dietas, não influenciaram o consumo de ração ao longo das 24 horas do dia. Conclui-se que os níveis de vitamina $E$ nas dietas, não influenciaram os padrões comportamentais e o consumo de ração no período de 24 horas dos frangos de corte aos 21 e 42 dias de idade.

Palavras-chave: Antioxidante; Nutriente funcional; Micronutrientes.

\section{ABSTRACT}

The objective of this study was to evaluate the effects of different levels of vitamin $E$ in broiler chickens on behavioral parameters and feed intake at different stages of production. 240 male broiler chicks, eight days old from the Cobb $500^{\circledR}$ strain, were used. The birds were homogenized and distributed in a completely randomized design, with four treatments (50, 100, 150 and 200\% of the vitamin E requirements), and six replications. The variables evaluated were the behavioral patterns of the birds, the feed consumption over 24 hours and the thermographic images. It was observed that the increasing levels of vitamin $E$ in the diets, did not influence the behavioral parameters, eating, drinking, leisure and other activities. There was no interaction between vitamin E levels for maximum, minimum surface temperatures and thermal amplitude, in the morning and afternoon. The levels of vitamin $E$ in the diets did not influence the consumption of feed throughout the 24 hours of the day. It was concluded that the levels of vitamin $E$ in the diets did not influence the behavioral patterns and feed intake in the 24-hour period of broilers at 21 and 42 days of age.

Keywords: Antioxidant; Functional nutrient; Micronutrients.

\section{RESUMEN}

El objetivo de este estudio fue evaluar los efectos de diferentes niveles de vitamina $E$ en pollos de engorde sobre los parámetros de comportamiento y el consumo de alimento en diferentes etapas de producción. Se utilizaron 240 pollos 
de engorde machos, con ocho días de vida de la cepa Cobb 500 ${ }^{\circledR}$. Las aves fueron homogeneizadas y distribuidas en un diseño completamente al azar, con cuatro tratamientos (50, 100, 150 y 200\% de los requerimientos de vitamina E), y seis repeticiones. Las variables evaluadas fueron los patrones de comportamiento de las aves, el consumo de alimento durante 24 horas y las imágenes termográficas. Se observó que los niveles crecientes de vitamina $E$ en las dietas no influían en los parámetros de comportamiento, alimentación, bebida, ocio y otras actividades. No hubo interacción entre los niveles de vitamina E para las temperaturas máximas, mínimas de la superficie y la amplitud térmica, en la mañana y la tarde. Los niveles de vitamina $E$ en las dietas no influyeron en el consumo de alimento durante las 24 horas del día. Se concluyó que los niveles de vitamina E en las dietas no influyeron en los patrones de comportamiento y el consumo de alimento en el período de 24 horas de los pollos de engorde a los 21 y 42 días de edad.

Descriptores: Antioxidante; Nutriente funcional; Micronutrientes.

\section{INTRODUÇÃO}

A termografia é uma técnica de processamento de imagem, utilizada para visualizar a temperatura superficial da pele ou objeto, medindo a emissão de energia infravermelha dentro de uma determinada faixa. A estimativa da temperatura da superfície da pele pode ser visualizada através de uma imagem numérica, referido como um termograma, composto por diferentes zonas de temperatura (NASCIMENTO et al., 2011).

Estudos têm demonstrado que frangos de corte criados em temperaturas elevadas, apresentam menor consumo de ração, menor ganho de peso e pior conversão alimentar. Nesse sentindo, fornecer alimentos que favoreça para menor produção de calor no processo de digestão e absorção, pode não comprometer o desempenho das aves, em situação de estresse por calor (VALÉRIO et al., 2003). Assim, avaliar o desempenho e o comportamento das aves suplementadas com vitamina $\mathrm{E}$ pode ser uma ferramenta útil para obtenção de dados sobre a expressão do animal em estresse por calor, estimando um importante parâmetro para avaliar a influência da dieta na produção e na sua qualidade de vida (PEREIRA; NÃÃS, 2005). A vitamina E possui ação antioxidante dos ácidos graxos não saturados, atua no metabolismo da célula e no metabolismo do ácido nucleico.

Desta forma, objetivou-se avaliar os efeitos de diferentes níveis de vitamina $\mathrm{E}$ na alimentação de DOI: http://dx.doi.org/10.20873/uftsuple2020-8600 frangos de corte sobre os parâmetros comportamentais e o consumo de ração em diferentes fases de produção.

\section{MATERIAIS E MÉTODOS}

O experimento foi conduzido no Setor de Avicultura da Escola de Medicina Veterinária e Zootecnia da Universidade Federal do Tocantins, aprovado pela Comissão de Ética no Uso de Animais da Universidade Federal do Tocantins (CEUA-UFT),

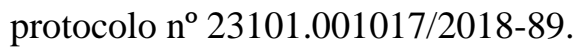

Foram utilizados 240 pintos de corte, machos, da linhagem Cobb $500^{\circledR}$, aos oito dias de idade, as aves foram homogeneizadas e distribuídas em delineamento experimental inteiramente casualizado (DIC), com quatro tratamentos $(50,100,150$ e $200 \%$ das exigências de vitamina $E$, de acordo com as recomendações de ROSTAGNO et al., 2017) e seis repetições. As aves foram alojadas em galpão experimental, provido de 24 boxes, com comedouros e bebedouros tubulares e pendulares, respectivamente.

$\mathrm{O}$ abastecimento dos comedouros e a limpeza dos bebedouros foram realizados duas vezes por dia.

Até o $14^{\circ}$ dia de vida, as aves foram aquecidas artificialmente, por lâmpadas incandescentes de 60 Ws. As condições ambientais no interior das instalações, foram monitoradas e registradas diariamente a cada 30 minutos, utilizando-se Data Loggers da marca HOBO ware OnSet ${ }^{\circledR}$ Versão 3.4.1. As aves foram alimentadas com dietas experimentais calculadas considerando as exigências nutricionais de

Revista Desafios -v. 7, Especial - PIBIC, 2020 
ROSTAGNO et al. (2017), nas fases de 1 a 7, de 8 a 21 e de 22 a 42 dias de idade.

As variáveis avaliadas foram os padrões comportamentais das aves (comendo, bebendo, ócio e outras atividades que são: (investigando penas, banho, comportamento agressivo, movimentos de conforto, agressividade, ciscando, sentada e parada)), o consumo de ração ao longo de 24 horas e as imagens termográficas. Para determinação dos parâmetros comportamentais foram selecionadas duas aves de cada unidade experimental, as quais foram marcadas com pintura no dorso.

As observações foram realizadas por filmagens, durante 10 minutos, em cada repetição, totalizando 60 minutos por tratamento, no período de $(7: 00-09: 00 h)$ e $(13: 00-16: 00 h)$ no final de cada de ciclo de vida das aves $\left(21^{\circ}\right.$ e $42^{\circ}$ dia). Após o término das filmagens em cada período, foram tiradas fotos, com câmera termográfica Flir E60®, com distância de mais ou menos $1 \mathrm{~m}$ das aves. Nos mesmos dias foram pesados os comedouros a cada 3 horas (07:00; 10:00; 13:00; 16:00; 19:00 e 07:00h), para avaliar o consumo de ração ao longo de 24 horas. Os padrões comportamentais foram adaptados segundo metodologia proposta por RUDKIN; STEWART (2003), levando-se em consideração as atividades desenvolvidas por cada ave.

As filmagens foram analisadas e calculadas as percentagens médias de tempo de expressão de cada comportamento. As imagens termográficas foram avaliadas com o auxílio do programa Flir Tools, para obtenção das temperaturas superficiais máxima, mínima e a amplitude térmica.

Os dados das variáveis avaliadas foram submetidos aos testes de Normalidade (Cramer Von Mises) e Homocedasticidade (Levene). Satisfeitas essas pressuposições, as variáveis foram submetidas à análise de variância. Adicionalmente as médias dos tratamentos foram comparadas pelo teste Student DOI: http://dx.doi.org/10.20873/uftsuple2020-8600
Newman Keuls (SNK) considerando um nível de significância igual ou inferior a 5\%. As análises estatísticas foram realizadas com o auxílio do programa estatístico SISVAR.

\section{RESULTADOS E DISCUSSÃO}

Observou-se que os níveis crescentes de vitamina $\mathrm{E}$ nas dietas, não influenciaram $(\mathrm{P}>0,05)$ os parâmetros comportamentais, comendo (COM), bebendo (BEB), ócio (OC) e outras atividades (OA) de frangos de corte aos 21 dias de idade (Figura 1).

Figura 1. Padrões comportamentais comendo (COM), bebendo (BEB), ócio e outras atividades (OAT) de frangos de corte (manhã e tarde) aos 21 dias de idade.

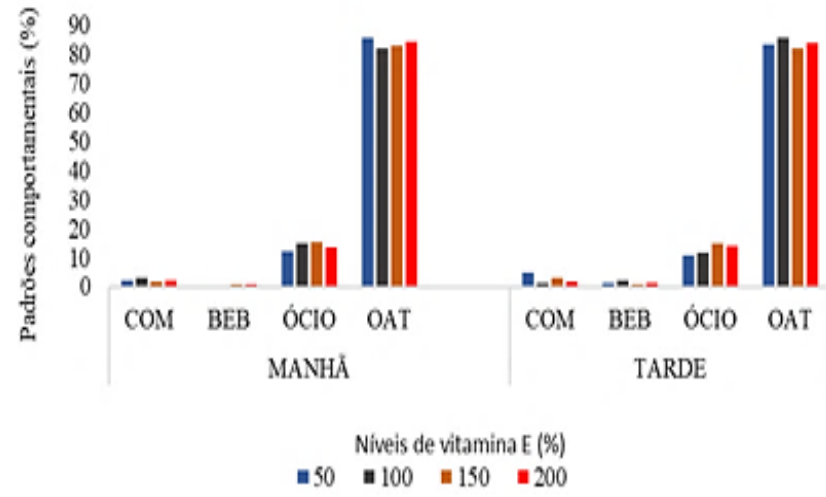

A temperatura ambiental média durante $\mathrm{o}$ período experimental foi de $34,7^{\circ} \mathrm{C}$, valor acima do considerado de conforto para as aves, no entanto, o valor da temperatura ambiental não alterou os padrões comportamentais, independentemente dos níveis de vitamina $\mathrm{E}$, o que pode ser justificado, pelo fato das aves terem sido criadas nas mesmas condições ambientais. Os níveis crescentes de vitamina E não influenciaram $\quad(\mathrm{p}>0,05) \quad$ os $\quad$ parâmetros comportamentais, comendo (COM), bebendo (BEB), ócio (OC) e outras atividades (OAT) de frangos aos 42 dias de idade (Figura 2).

Revista Desafios -v. 7, Especial - PIBIC, 2020 
Figura 2. Padrões comportamentais comendo (COM), bebendo (BEB), ócio e outras atividades de frangos de corte (manhã e tarde) aos 42 dias de idade.

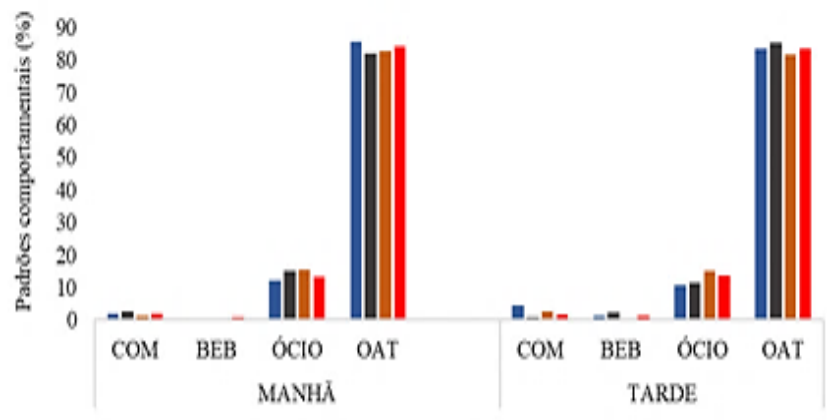

Niveis de vitamina $\mathrm{E}(\%)$

- $50 \quad 100 \quad 150 \quad 200$
Não houve interação entre os níveis de vitamina $E$ nas dietas $(P>0,05)$ para as temperaturas superficiais máxima, mínima e a amplitude térmica, nos períodos da manhã e tarde.

No entanto, as temperaturas máximas, mínimas e a amplitude térmica foram influenciadas $(\mathrm{P}<0,05)$ pelos períodos, com menores temperaturas, para o período da manhã (Tabela 1).

Tabela 1. Valores das temperaturas superficiais máximas $\left({ }^{\circ} \mathrm{C}\right)$, mínimas $\left({ }^{\circ} \mathrm{C}\right)$ e amplitude térmica $\left({ }^{\circ} \mathrm{C}\right)$ de frangos de corte aos 21 dias, alimentados com diferentes níveis de vitamina E.

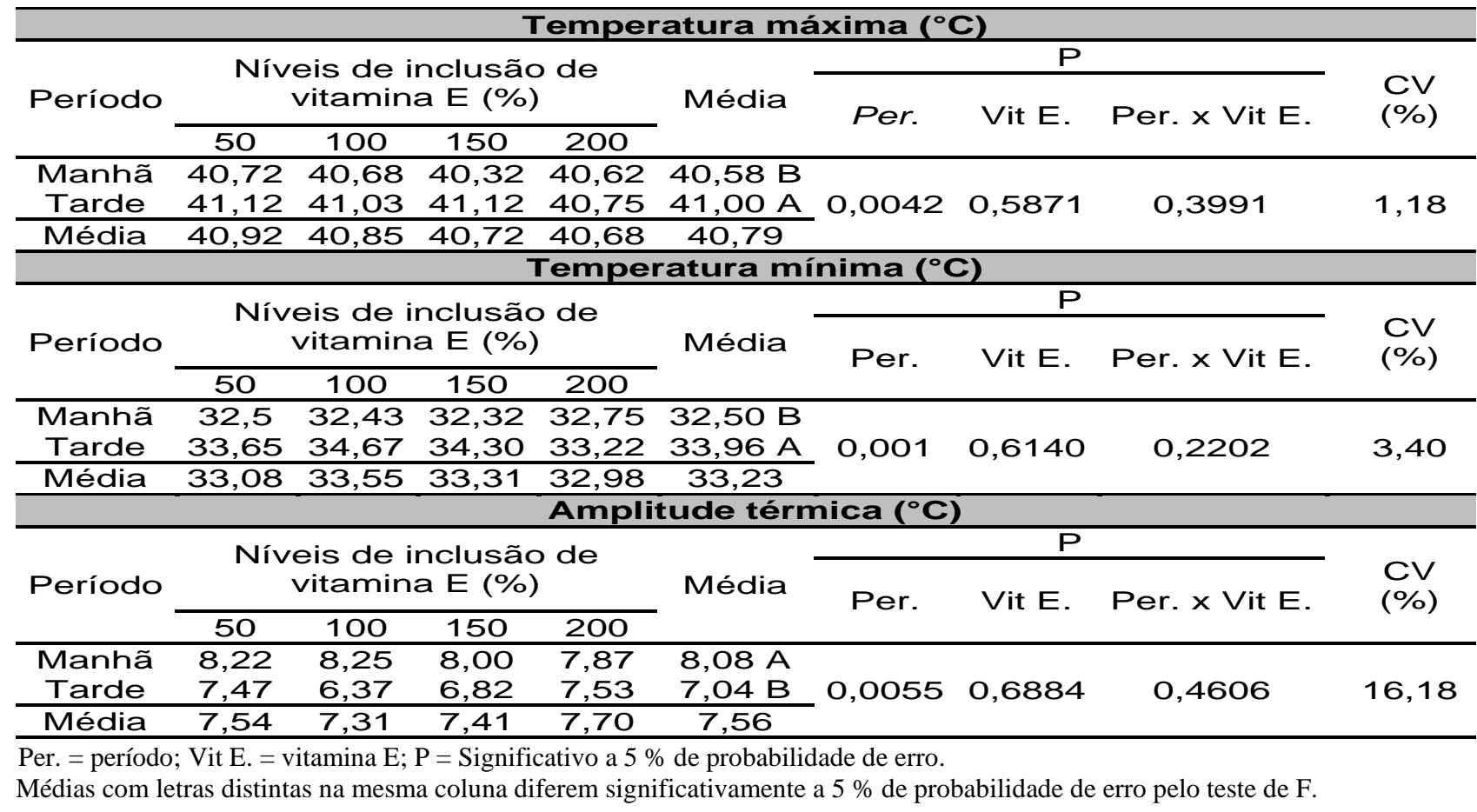

Não houve interação entre os níveis de vitamina E nas dietas $(\mathrm{P}>0,05)$ para as temperaturas máxima, mínima e a amplitude térmica, nos períodos da manhã e tarde. No entanto, as temperaturas máximas, mínimas e amplitude térmica foram influenciadas $(\mathrm{P}<0,05)$ pelos períodos aos 42 dias de idade, com menores temperaturas. para o período da manhã (Tabela 2).

As condições ambientais dentro do sistema de criação influenciam diretamente na condição de conforto térmico e bem-estar, afetando a temperatura superficial das aves (NAZARENO et al., 2009).

As diferenças de temperaturas superficiais máxima e mínima das aves nos períodos de manhã e tarde, podem estar associadas aos valores das temperaturas dentro das instalações nesses horários, que foram de 27,3 e $33,3{ }^{\circ} \mathrm{C}$, sendo o ITGU de 74,1 e 82,1 respectivamente. 
Tabela 2. Valores das temperaturas superficiais máximas $\left({ }^{\circ} \mathrm{C}\right)$, mínimas $\left({ }^{\circ} \mathrm{C}\right)$, amplitude térmica $\left({ }^{\circ} \mathrm{C}\right)$ de frangos de corte aos

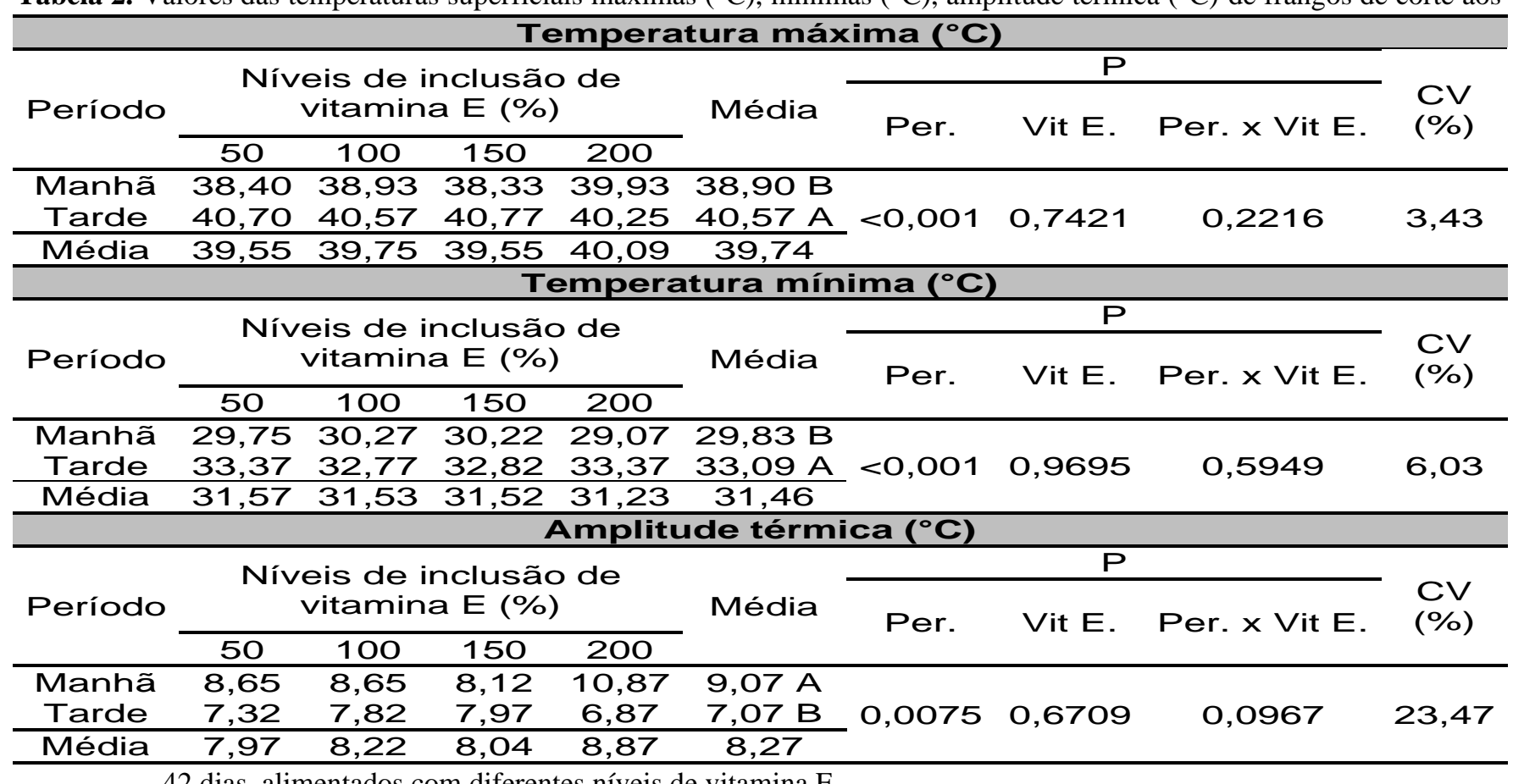

42 dias, alimentados com diferentes níveis de vitamina $\mathrm{E}$.

Per. = período; Vit E. = vitamina $\mathrm{E} ; \mathrm{P}=$ Significativo a $5 \%$ de probabilidade de erro.

Médias com letras distintas na mesma coluna diferem significativamente a $5 \%$ de probabilidade de erro pelo teste de F.

Observou-se que os níveis de vitamina E nas dietas, não influenciaram $(\mathrm{P}>0,05)$ o consumo de ração de frangos de corte ao longo das 24 horas do dia, das 07:00 às 19:00 h, e das 19:00 às 07:00 h, aos 21 e os 42 dias de idade (Tabela 3).

Quando submetidos a temperaturas acima da zona de conforto térmico as aves tendem a reduzir o consumo de ração, na tentativa de minimizar a produção de calor metabólico, pela dificuldade em trocar calor com ambiente, o que consequentemente, reduz o desempenho produtivo (BROSSI et al., 2009).

Tabela 3. Valores médios de consumo de ração (g) ao longo de 24 horas de frangos de corte aos 21 e 42 de idade. ${ }^{1}$ Coeficiente de variação $(\%)$.

\begin{tabular}{|c|c|c|c|c|c|c|}
\hline \multirow[b]{2}{*}{ Horários } & \multicolumn{4}{|c|}{ Níveis de vitamina E $(\%)$} & \multirow{2}{*}{$C^{1}$} & \multirow{2}{*}{$P>F^{2}$} \\
\hline & 50 & 100 & 150 & 200 & & \\
\hline \multicolumn{7}{|c|}{21 dias } \\
\hline 07:00 -10:00 & 33,04 & 32,83 & 32,54 & 33,72 & 10,18 & 0,9383 \\
\hline $10: 00-13: 00$ & 16,75 & 18,67 & 17,26 & 16,30 & 16,14 & 0,4995 \\
\hline 13:00 - 16:00 & 19,04 & 19,33 & 21,20 & 21,96 & 17,61 & 0,4389 \\
\hline 16:00 - 19:00 & 15,25 & 17,33 & 15,80 & 14,06 & 13,97 & 0,1046 \\
\hline 19:00 - 07:00 & 103,88 & 100,17 & 99,24 & 101,56 & 7,08 & 0,7036 \\
\hline \multicolumn{7}{|c|}{42 dias } \\
\hline $07: 00-10: 00$ & 37,67 & 35,00 & 31,91 & 34,11 & 10,86 & 0,0977 \\
\hline $10: 00-13: 00$ & 20,75 & 23,33 & 22,43 & 24,78 & 16,37 & 0,3271 \\
\hline 13:00 - 16:00 & 26,88 & 25,00 & 24,98 & 24,98 & 21,43 & 0,8983 \\
\hline 16:00 - 19:00 & 17,17 & 17,83 & 17,48 & 18,45 & 17,07 & 0,8975 \\
\hline 19:00 - 07:00 & 120,70 & 122,50 & 117,20 & 114,19 & 7,31 & 0,3762 \\
\hline
\end{tabular}




\section{CONCLUSÃO}

Conclui-se que os níveis de vitamina $\mathrm{E}$ nas dietas, não influenciaram os padrões comportamentais e o consumo de ração dos frangos de corte aos 21 e aos 42 dias de idade.

\section{AGRADECIMENTO}

O presente trabalho foi realizado com o apoio do Conselho Nacional de Desenvolvimento Científico e Tecnológico - CNPq - Brasil.

Todos os autores declararam não haver qualquer potencial conflito de interesses referente a este artigo.

\section{REFERÊNCIAS}

BROSSI, C.; CONTRERAS-CASTILLO, C. J.; AMAZONAS, E. D. A.; e MENTEN, J. F. M. Estresse térmico durante o pré-abate em frangos de corte. Ciência Rural, Santa Maria, v. 39, n. 4, p. 1284-1293, 2009.

NASCIMENTO, G.R.do; PEREIRA, D.F.; NÄÄS, I.A.; RODRIGUES, L.H.A. Índice fuzzy de conforto térmico para frangos de corte. Engenharia Agrícola, v.31, n.2, p.219-229, 2011.

NAZARENO, A. C., PANDORFI, H., ALMEIDA, G. L., GIONGO, P. R., PEDROSA, E. M., GUISELINI, C. Avaliação do conforto térmico e desempenho de frangos de corte sob regime de criação diferenciado. Revista Brasileira de Engenharia Agrícola e Ambiental, v. 13, n. 6, p. 802-808, 2009.

PEREIRA, D.F.; NÄÄS, I.A. Estimativa do conforto de matrizes de frango de corte baseada em análise do comportamento de preferência térmica. Engenharia Agrícola, Jaboticabal, v.25, n.2, p. 315-321, 2005.
ROSTAGNO, H. S.; ALBINO, L. F. T.; DONZELE, J. L. GOMES, P. C.; OLIVEIRA, R. F.; LOPES, D. C.; FERREIRA, A. S. Composição de alimentos e exigências nutricionais. Tabelas Brasileiras para aves e suínos. Viçosa, MG: UFV, Departamento de Zootecnia, p. 141, 2017.

RUDKIN, C.; STEWART, G.D. Behaviour of hens in cages - A pilot study using video tapes. A report for the rural industries research and development corporation, v.40, n.477, p.102, 2003.

VALÉRIO, S.R.; OLIVEIRA, R.F.M.; DONZELE, J.L.; GOMES, P.C.; APOLÔNIO, L.R.; RESENDE, W.O. Níveis de lisina digestível em rações, em que se manteve ou não a relação aminoacídica, para frangos de corte de 22 a 42 dias de idade, mantidos em estresse por calor. Revista Brasileira de Zootecnia, v.32, n.2, p.372-382, 2003. 